\title{
ON THE FIRST $n$ STRONGLY COMPACT CARDINALS
}

\author{
ARTHUR W. APTER
}

(Communicated by Andreas R. Blass)

\begin{abstract}
Using techniques of Kimchi and Magidor, we generalize an earlier result and show that it is relatively consistent for the first $n$ strongly compact cardinals to be somewhat supercompact yet not fully supercompact.
\end{abstract}

The class of strongly compact cardinals is, without a doubt, one of the most peculiar in the entire theory of large cardinals. As is well known, the class of strongly compact cardinals suffers from a severe identity crisis. Magidor's fundamental result of [Ma] shows that it is consistent for the least strongly compact cardinal to coincide with either the least measurable or the least supercompact cardinal. The result of [A1] shows that it is consistent for the least strongly compact cardinal to be somewhat supercompact although not fully supercompact. The result of Kimchi and Magidor [KM] shows that it is consistent for the class of strongly compact cardinals to coincide with the class of supercompact cardinals (except at limit points where Menas' result [Me] shows that such a coincidence may not occur) or for the first $n$ for $n \in \omega$ strongly compact cardinals to coincide with the first $n$ measurable cardinals.

The purpose of this paper is to show that matters can be muddled still further. Specifically, we generalize the result of [A1] using the methods of [KM] and prove the following

Theorem. Let $V \vDash " K=\left\{\kappa_{1}, \ldots, \kappa_{n}\right\}$ with $\kappa_{1}<\kappa_{2}<\cdots<\kappa_{n}$ the first $n$ supercompact cardinals". For each $\kappa \in K$, let $\varphi_{\kappa}$ be a formula in the language of set theory which defines an increasing $\Sigma_{2}$ function from the ordinals to the ordinals which, in addition, has the following properties:

(a) $\varphi_{\kappa}$ is preserved at and above $\kappa$ when forcing with a cardinal preserving partial ordering of size $\kappa$, i.e., for $P \in V$ a cardinal preserving partial ordering, $|P|=\kappa, \alpha \geq \kappa, V \vDash " \delta=\varphi_{\kappa}(\alpha)$ " iff $V^{P} \vDash " \delta=\varphi_{\kappa}(\alpha)$ ".

(b) If for any model $M$ of $Z F C, M \vDash " \alpha<\beta$ and $\beta$ is $\varphi_{\kappa}(\beta)$ supercompact", then $\varphi_{\kappa}(\alpha)<\beta$.

(c) If for any model $M$ of $Z F C, M \vDash " \delta$ is $\varphi_{\kappa_{i}}(\delta)$ supercompact, $\delta$ is $\varphi_{\kappa_{j}}(\delta)$ supercompact, and $i \leq j$ ", then $\varphi_{\kappa_{i}}(\delta) \geq \varphi_{\kappa_{j}}(\delta)$.

There is then a partial ordering $P$ so that for each $\kappa \in K, V^{P} \vDash$ “ $\kappa$ is

Received by the editors March 14, 1991 and, in revised form, November 29, 1993.

1991 Mathematics Subject Classification. Primary 03E35, 03E55.

The research for this paper was partially supported by PSC-CUNY Grants 661371 and 662341 and by a salary grant from Tel Aviv University. 
at least $\varphi_{\kappa}(\kappa)$ supercompact, $\kappa$ is not supercompact, and $\kappa$ is fully strongly compact"; further, $V^{P} \vDash$ " $K$ consists of the first $n$ strongly compact cardinals".

Restrictions (a) and (b) above on each $\varphi_{\kappa}$ are made for the same technical reasons as in [A1]. Note, however, that many $\Sigma_{2}$ functions, e.g., $\delta \mapsto \delta^{+}$, $\delta \mapsto$ The least inaccessible $>\delta, \delta \mapsto$ The least measurable $>\delta$, etc., meet restrictions (a) and (b). Restriction (c) is needed to make the arguments of $[\mathrm{KM}]$ go through and will be explained at the end of the paper.

Our Theorem essentially says that it is possible for the first $n$ strongly compact cardinals to be somewhat supercompact yet not fully supercompact. This provides an intermediate result to the results of [KM], which tell us that the first $n$ strongly compact cardinals can be either the first $n$ measurable cardinals or the first $n$ supercompact cardinals.

Before beginning the proof of our Theorem, we briefly mention some preliminary material. Essentially, our notation is standard, with $V_{\alpha}$ representing the universe through stage $\alpha$, and for $\alpha<\beta$ ordinals, $[\alpha, \beta],[\alpha, \beta),(\alpha, \beta]$, and $(\alpha, \beta)$ as in standard interval notation. Further, we assume complete familiarity with the notions of strong compactness, supercompactness, etc. [A1], [A2], [KM], [L], [Ma], [Me], and [SRK] will provide sufficient details. Finally, we will assume some familiarity with the techniques and methods of [KM], although where necessary, appropriate details will be provided.

We turn now to the proof of our Theorem.

Proof of Theorem. We will first define, for each $\kappa \in K$, a partial ordering $P^{\kappa}$ so that $V^{P^{\kappa}} \vDash " \kappa$ is at least $\varphi_{\kappa}(\kappa)$ supercompact, $\kappa$ is not supercompact, $\kappa$ is fully strongly compact, and there are no strongly compact cardinals in the interval $\left[\delta_{\kappa}, \kappa\right) "$, where $\delta_{\kappa_{1}}=\aleph_{1}$ and $\delta_{\kappa_{i}}=\kappa_{i-1}^{+}$if $2 \leq i \leq n$. Then the partial ordering $P$ used to construct our final model will be the product ordering $\prod_{1<i<n} P^{\kappa_{i}}$.

To define $P^{\kappa}$, we proceed inductively. $P_{0}^{\kappa}$ is the trivial partial ordering, and if $\lambda<\kappa$ is a limit ordinal, then $P_{\lambda}^{\kappa}=$ inverse limit $\left(\left\langle P_{\alpha}^{\kappa}: \alpha<\lambda\right\rangle\right)$ if $\lambda$ is singular, and $P_{\lambda}^{\kappa}=$ direct limit $\left(\left\langle P_{\alpha}^{\kappa}: \alpha<\lambda\right\rangle\right)$ if $\lambda$ is regular. To define $P_{\alpha+1}^{\kappa}$, let $\gamma_{\alpha}<\kappa$ be the least ordinal so that $\Vdash_{P_{\alpha}}$ " $\gamma_{\alpha}$ is $\varphi_{\kappa}\left(\gamma_{\alpha}\right)$ supercompact". $\gamma_{\alpha}$ will exist, since $\kappa$ is supercompact and $\varphi_{\kappa}$ is $\Sigma_{2} . \dot{Q}_{\alpha}^{\kappa}$ is then a term for the partial ordering $Q_{\alpha}^{\kappa} \in V^{P_{\alpha}^{\kappa}}$ which adds a non-reflecting stationary set of ordinals of cofinality $\delta_{\kappa}$ to $\gamma_{\alpha}$; specifically, $\dot{Q}_{\alpha}^{\kappa}$ is a term for a non-reflecting bounded non-stationary subset of $\left\{\beta<\gamma_{\alpha}: \operatorname{cof}(\beta)=\delta_{\kappa}\right\}$, ordered by $q$ extends $p$ iff $q \supseteq p$ and $q$ is an end extension of $p$, i.e., $p=q \cap \sup (p) . P_{\alpha+1}^{\kappa}$ is then defined as $P_{\alpha}^{\kappa} * \dot{Q}_{\alpha}^{\kappa}$, and $P^{\kappa}=\operatorname{direct}$ limit $\left(\left\langle P_{\alpha}^{\kappa}: \alpha<\kappa\right\rangle\right)$.

Lemma 1. $V^{P^{\kappa}} \vDash " \kappa$ is strongly compact and there are no strongly compact cardinals in the interval $\left[\delta_{\kappa}, \kappa\right)$ ".

Proof of Lemma 1. As in [KM], forcing with $P^{\kappa}$ or any of its component partial orderings will create no new measurable cardinals; in fact, the arguments of [KM] show that forcing with $P^{\kappa}$ or any of its component partial orderings will create no new cardinals $\delta$ which are $\varphi_{\kappa}(\delta)$ supercompact. Thus, since the cardinals $\delta$ in the field of $P^{\kappa}$ are all forced by some component of $P^{\kappa}$ to be $\varphi_{\kappa}(\delta)$ supercomponent, each cardinal $\delta$ in the field of $P^{\kappa}$ will be $\varphi_{\kappa}(\delta)$ supercompact in $V$. Thus, the arguments of [KM] show that $V^{P^{\kappa}} \vDash$ " $\kappa$ is 
strongly compact", assuming there are only finitely many cardinals $\delta>\kappa$ so that $V \vDash$ " $\delta$ is $\varphi_{\kappa}(\delta)$ supercompact". That we can make this assumption follows from restriction (c) of the Theorem and the definition of $P$, since restriction (c) ensures the portion of the forcing $P$ above $P^{\kappa}$ leaves only finitely many $\delta>\kappa$ so that $\delta$ is $\varphi_{\kappa}(\delta)$ supercompact. We will comment upon this further in the proof of Lemma 4 and at the end of the paper. We mention now, however, that if there are any cardinals $\delta$ above the largest cardinal $\kappa_{n}$ so that $\delta$ is $\varphi_{\kappa_{n}}(\delta)$ supercompact, then we either have to cut the universe off at the least such $\delta$ or else iterate the forcing $P^{\kappa_{n}}$ above $\kappa_{n}$ to destroy all such $\delta$ if the strong compactness of $\kappa_{n}$ is to be preserved.

To show that $V^{P^{\kappa}} \vDash$ "There are no strongly compact cardinals in the interval $\left[\delta_{\kappa}, \kappa\right)$ ", it suffices to show that for unboundedly many $\gamma \in\left[\delta_{\kappa}, \kappa\right), V^{P^{\kappa}} \vDash$ "There is a non-reflecting stationary subset of $\gamma$ of ordinals of cofinality $\delta_{\kappa}$ ". (This is since a theorem of [SRK] states that if $\beta$ contains a non-reflecting stationary subset of ordinals of cofinality $\alpha$, then there are no strongly compact cardinals in the interval $(\alpha, \beta)$. Here $\delta_{\kappa}$, being a successor cardinal, of course isn't strongly compact.) To see this, let $\gamma$ be a cardinal in the field of $P^{\kappa}$, and let $P^{\kappa}=Q_{\gamma} * \dot{Q}^{\gamma}$, where the field of $Q_{\gamma}$ consists of all cardinals $\leq \gamma$ and the field of $\dot{Q}_{\gamma}$ consists of all cardinals $>\gamma$. By the definition of $P^{\kappa}, V^{Q_{\gamma}} \vDash^{\text {" }} \gamma$ contains a non-reflecting stationary subset of ordinals of cofinality $\delta_{\kappa}$ ". The definition of $P^{\kappa}$ ensures that for any $\alpha$ so that $P^{\kappa}=P_{\alpha}^{\kappa} * \dot{Q}_{\alpha}^{\kappa}$ and $\Vdash_{P_{\alpha}^{\kappa}}$ "The field of $\dot{Q}_{\alpha}^{\kappa}$ is a $V$ measurable cardinal $>\gamma$ ", $\Vdash_{P_{\alpha}^{\kappa}}$ “ $\dot{Q}_{\alpha}^{\kappa}$ is $<\sigma_{\gamma}$ strategically closed" where $\sigma_{\gamma}$ is the least (measurable) cardinal in the field of $\dot{Q}^{\gamma}$ and $<\sigma_{\gamma}$ strategically closed for a partial ordering $Q$ means that for any fixed $\beta<\sigma_{\gamma}$, in the two person game in which the players construct a sequence $\left\langle q_{\alpha}: \alpha<\beta\right\rangle$ so that each $q_{\alpha_{0}}$ extends $q_{\alpha}$ for $\alpha<\alpha_{0}$ and player I plays odd stages and player II plays even and limit stages, player II always has a winning strategy ensuring that the game can be continued for any $\alpha<\beta$. Since each cardinal $\alpha$ in the field of $\dot{Q}^{\gamma}$ is a $V$ measurable cardinal so that $\alpha \geq \sigma_{\gamma}>\gamma$, the definition of $\dot{Q}^{\gamma}$ ensures that $\Vdash_{Q_{\gamma}}$ " $\dot{Q}^{\gamma}$ is $<\sigma_{\gamma}$ directed closed", i.e., that $\Vdash_{Q_{\gamma}}$ "Forcing with $\dot{Q}^{\gamma}$ adds no new subsets of $\gamma$ ". This means that $V^{Q_{\gamma} * \dot{Q}^{\gamma}}=V^{P^{\kappa}} \vDash$ " $\gamma$ contains a non-reflecting stationary subset of ordinals of cofinality $\delta_{\kappa} "$. Since there are unboundedly many such $\gamma \in\left[\delta_{\kappa}, \kappa\right)$ in the field of $P^{\kappa}$, Lemma 1 is proved.

Lemma 2. $V^{P^{\kappa}} \vDash$ " $\kappa$ is not supercompact".

Proof of Lemma 2. If $V^{P^{\kappa}} \vDash$ " $\kappa$ is supercompact", let $j$ be a supercompact embedding of $V^{P^{\kappa}}$ into a sufficiently closed inner model $M$ with critial point $\kappa$ so that $M \vDash$ " $\kappa$ is $\varphi_{\kappa}(\kappa)$ supercompact". By reflection, $A=\{\gamma<\kappa: \gamma$ is $\varphi_{\kappa}(\gamma)$ supercompact $\}$ is unbounded in $\kappa$. It is also the case that $M \vDash$ " $\kappa$ is a stage in the definition of $j\left(P^{\kappa}\right)$ at which a (direct) limit is taken", so reflection again allows us to assume that for any $\gamma \in A, P_{\gamma}^{\kappa}=$ (direct) limit $\left(\left\langle P_{\alpha}^{\kappa}: \alpha<\gamma\right\rangle\right)$. By the definition of $P^{\kappa}, P_{\gamma+1}^{\kappa}=P_{\gamma}^{\kappa} * \dot{Q}_{\gamma}^{\kappa}$ is so that $\Vdash_{P_{\gamma+1}^{\kappa}}{ }^{\text {" }} \gamma$ contains a non-reflecting stationary subset of ordinals of cofinality $\delta_{\kappa}$, , so the proof of Lemma 1 tells us that this fact is also true in $V^{P^{\kappa}}$, i.e., that $V^{P^{\kappa}} F^{\text {“ } \gamma} \gamma$ is not $\varphi_{\kappa}(\gamma)$ supercompact"; in fact, $V^{P^{\kappa}} \vDash$ " $\gamma$ isn't weakly compact". This contradiction proves Lemma 2.

Lemma 3. $V^{P^{\kappa}} \vDash " \kappa$ is $\varphi_{\kappa}(\kappa)$ supercompact". 
Proof of Lemma 3. Since $\varphi_{\kappa}$ is $\Sigma_{2}$, we can find a supercompact embedding $j: V \rightarrow M$ with critical point $\kappa$ so that $\varphi_{\kappa}(\kappa)^{M}=\varphi_{\kappa}(\kappa)^{V}$. Further, the embedding $j$ can be chosen so that $M$ is at least $2^{\left[\varphi_{\kappa}(\kappa)\right]^{<\kappa}}$ closed. Property (a) of $\varphi_{\kappa}$ of the hypotheses of the Theorem shows that $\varphi_{\kappa}(\kappa)$ has the same meaning in either $V^{P^{\kappa}}$ or $M^{P^{\kappa}}$ as it did in $V$ or $M$. We can therefore write $\varphi_{\kappa}(\kappa)$ unambiguously.

If $V^{P^{\kappa}} \vDash$ " $\kappa$ is $\varphi_{\kappa}(\kappa)$ supercompact", then we are done, so assume this is not the case, i.e., $V^{P^{\kappa}} F$ " $\kappa$ is not $\varphi_{\kappa}(\kappa)$ supercompact". Work now in $M$. $P^{\kappa}$ is an initial segment of $j\left(P^{\kappa}\right)$, and by the closure properties of $M, M^{P^{\kappa}} \vDash$ " $\kappa$ is not $\varphi_{\kappa}(\kappa)$ supercompact". Writing $j\left(P^{\kappa}\right)$ as $P^{\kappa} * \dot{Q}$, property (b) of $\varphi_{\kappa}$ of the hypotheses of the Theorem allows us to conclude that for the least cardinal $\sigma_{\kappa}$ in the field of $\dot{Q}, M^{P^{\kappa}} \vDash " \varphi_{\kappa}(\kappa)<\sigma_{\kappa}$ ".

If $G$ is $V$-generic over $P^{\kappa}$ and $H$ is $M[G]$-generic over $Q$, define an embedding $j^{*}: V[G] \rightarrow M[G * H]$ by $j^{*}\left(i_{G}(\tau)\right)=i_{G * H}(j(\tau))$ for any term $\tau$ denoting a set in $V[G]$. Since $\varphi_{\kappa}(\kappa)<\sigma_{\kappa}$, the closure properties of $M$ and the Kunen-Paris arguments of [KP] show that $j^{*}$ is a well-defined elementary embedding with criticial point $\kappa$ so that the ultrafilter $\mathscr{U}$ defined by $x \in \mathscr{U}$ iff $\left\langle j(\alpha): \alpha\left\langle\varphi_{\kappa}(\kappa)\right\rangle \in j^{*}(x)\right.$ is a supercompact ultrafilter over $P_{\kappa}\left(\varphi_{\kappa}(\kappa)\right)^{V[G]}$ present in $V[G * H]$. In $M[G]$, since $\varphi_{\kappa}(\kappa)<\sigma_{\kappa}$ and $\sigma_{\kappa}$ is inaccessible, $2^{\left[\varphi_{\kappa}(\kappa)\right]^{<\kappa}}<\sigma_{\kappa}$, meaning that $Q$ will be so that forcing with $Q$ over $M[G]$ adds no new subsets of $2^{\left[\varphi_{\kappa}(\kappa)\right]^{<\kappa}}$. The closure properties of $M$ ensure that this property is true as well when forcing with $Q$ over $V[G]$, i.e., that $\mathscr{U} \in V[G]$. Thus, assuming $\Vdash_{P \kappa}$ " $\kappa$ is not $\varphi_{\kappa}(\kappa)$ supercompact" leads to the contradiction $\Vdash_{P \kappa}$ " $\kappa$ is $\varphi_{\kappa}(\kappa)$ supercompact" as well, meaning it must be the case that $\mathbb{1}_{P \kappa}$ " $\kappa$ is $\varphi_{\kappa}(\kappa)$ supercompact". This proves Lemma 3.

Lemma 4. $V^{P} \vDash$ "If $\kappa \in K$, then $\kappa$ is strongly compact, at least $\varphi_{\kappa}(\kappa)$ supercompact, but not fully supercompact".

Proof of Lemma 4. For each $\kappa \in K$, write $P=Q_{\kappa} \times Q^{\kappa}$, where $Q_{\kappa}=$ $\prod_{\{\lambda \in K: \lambda \leq \kappa\}} P^{\lambda}$ and $Q^{\kappa}=\prod_{\{\lambda \in K: \lambda>\kappa\}} P^{\lambda}$; further, write $Q_{\kappa}$ as $Q_{<\kappa} \times P^{\kappa}$ for $Q_{<\kappa}=\prod_{\{\lambda \in K: \lambda<\kappa\}} P^{\lambda}$. It is a fundamental result of both [A2] and [KM] that we can assume that a preliminary forcing has been done to ensure that $V \vDash$ "If $\kappa$ is a supercompact cardinal, then $\kappa$ is Laver [L] indestructible". Thus, since each component partial ordering $P^{\lambda}$ of $Q^{\kappa}$ has been defined so as to be at least $\kappa$-directed closed, $Q^{\kappa}$ is $\kappa$-directed closed, so $V Q^{\kappa} \vDash$ " $\kappa$ is a supercompact cardinal". As the subsets of $\kappa$ are the same in either $V$ or $V^{Q^{*}}$, the definition of $P^{\kappa}$ is the same in either $V$ or $V^{Q^{*}}$, so by Lemmas 1-3, since restriction (c) ensures $V^{Q_{\kappa}} \vDash$ "There are only finitely many $\delta>\kappa$ which are $\varphi_{\kappa}(\delta)$ supercompact", $V Q^{\kappa} \times P^{\kappa} \vDash " \kappa$ is strongly compact, at least $\varphi_{\kappa}(\kappa)$ supercompact, but not fully supercompact". Since $K$ is a finite set, the fact that forcing with $P^{\kappa}$ over either $V$ or $V Q^{\alpha}$ adds no new bounded subsets to $\kappa$ ensures that $V Q^{\kappa} \times P^{\kappa} \vDash "\left|Q_{<\kappa}\right|<\kappa$ ", so the Lévy-Solovay results [LS] ensure that $V^{Q^{\kappa} \times P^{\kappa} \times Q_{<\kappa}}=V^{P} \vDash " \kappa$ is strongly compact, at least $\varphi_{\kappa}(\kappa)$ supercompact, but not fully supercompact". This proves Lemma 4 .

Lemma 5. $V^{P} \vDash$ " $K$ consists of the first $n$ strongly compact cardinals".

Proof of Lemma 5. Assume that $V^{P} \vDash$ " $\gamma$ is the $i$ th strongly compact cardinal for $1 \leq i \leq n$ and isn't an element of $K$ ". As Lemma 4 tells us each $\kappa \in K$ 
remains strongly compact in $V^{P}$, it must be the case that $\gamma<\kappa_{n}$. Thus, if $\kappa$ is the least element of $K>\gamma$, then using the same notation as before, $\gamma \in\left[\delta_{\kappa}, \kappa\right)$. Writing $P=Q^{\kappa} \times P^{\kappa} \times Q_{<\kappa}$ as in the preceding lemma, the closure properties of $Q^{\kappa}$ already noted and Lemma 1 show that $V^{Q^{\kappa} \times P^{\kappa}} \vDash$ " $\gamma$ is not strongly

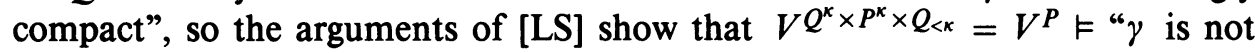
strongly compact". This proves Lemma 5.

Lemmas 1-5 complete the proof of our Theorem.

We note that each $\varphi_{\kappa}$ may imply additional assumptions on $\kappa$, e.g., if $\varphi_{\kappa}$ is the function $\delta \mapsto$ The least inaccessible cardinal $>\delta$, then there is assumed to be (if it can't already be shown to exist) an inaccessible cardinal $>\kappa$. Also, in general, for the same reasons as in [A1], $\varphi_{\kappa}$ can't be assumed to be $\Sigma_{3}$. Further, as in [A1], under certain circumstances we can get a precise bound on the non-supercompactness of some $\kappa \in K$. If, for instance, $\varphi_{\kappa}$ is the function $\delta \mapsto \delta^{+}$, then the argument of [L] or [KM] allows us to assume without loss of generality that $2^{\kappa}=\kappa^{+}$and $2^{\kappa^{+}}=\kappa^{++}$. After applying the arguments of Lemmas $2-4, \kappa$ will be $\kappa^{+}$supercompact but not $\kappa^{++}$supercompact. (Even if we have no knowledge of the size of power sets of cardinals, for many $\varphi_{\kappa}$ the preceding arguments show that $\kappa$ isn't $2^{\left[\varphi_{\kappa}(\kappa)\right]^{<\kappa}}$ supercompact.)

We remark that in the initial version of this paper, our Theorem was stated for a class of strongly compact cardinals and not just for a finite set. Unfortunately, as was pointed out by the referee, there is a gap in the original Kimchi-Magidor proof so that it is now only known how, from the existence of $n \in \omega$ supercompact cardinals, to force and obtain the consistency of the coincidence of the first $n$ strongly compact cardinals with the first $n$ measurable cardinals. To outline the original Kimchi-Magidor argument and highlight the problem, let $K=\left\{\kappa_{1}, \ldots, \kappa_{n}\right\}$ be as in the statement of our Theorem, and for each $\kappa \in K$, let $A_{\kappa} \subseteq \kappa-\{\delta \in K: \delta<\kappa\}$ be a set of measurable cardinals. Let $P^{\kappa}$ be defined as before, only this time adding non-reflecting stationary sets to the elements of $A_{\kappa}$, and again let $P=\prod_{1 \leq i \leq n} P^{\kappa_{i}}$.

To show $V^{P} \vDash$ " $\kappa$ is strongly compact for $\kappa \in K$ ", as in Lemma 4, it will suffice to show $V^{P^{\kappa}} \vDash$ " $\kappa$ is strongly compact". To do this, let $j: V \rightarrow M$ be an elementary embedding witnessing $\kappa$ is $\lambda$ strongly compact. If $G$ is $V$-generic over $P^{\kappa}$, then as usual, we'd like to find $H \subseteq j\left(P^{\kappa}\right), H \in V[G]$ so that $G * H$ is $M$-generic over $j\left(P^{\kappa}\right)$ and so that $j$ extends to a strongly compact embedding $j^{\prime}: V[G] \rightarrow M[G * H]$. If $\lambda$ is sufficiently large, then if $j$ is a $\lambda$ supercompact embedding and $\kappa_{i+1}, \ldots, \kappa_{n} \in j\left(A_{\kappa}\right)$ (as would certainly be the case in the original Kimchi-Magidor situation, i.e., when all ground model measurable cardinals are being destroyed), by elementariness, we would necessarily have $M[G * H] \vDash$ " $\kappa_{i+1}, \ldots, \kappa_{n}$ are no longer measurable as they contain non-reflecting stationary sets". By the closure properties of $M$, this would also have to be true in $V\left[G * H^{\prime}\right]$ for $H^{\prime}$ some "initial segment" of $H$, and we'd actually have to extend $V$ by $G * H^{\prime}$ and have $j^{\prime}$ be so that $j^{\prime}: V\left[G * H^{\prime}\right] \rightarrow M[G * H]$. This would mean that $\kappa_{i+1}, \ldots, \kappa_{n}$ could no longer be strongly compact in $V\left[G * H^{\prime}\right]$.

To avoid this difficulty, Kimchi and Magidor construct a strongly compact embedding $j: V \rightarrow M$ so that $M \vDash$ " $\kappa_{i+1}, \ldots, \kappa_{n}$ are non-measurable". They do this inductively as follows. Let $\lambda$ be sufficiently large, and let $j_{i}: V \rightarrow M_{i+1}$ be an embedding witnessing the $\lambda$ supercompactness of $\kappa$ so that $\kappa_{i+1}, \ldots, \kappa_{n}$ 
$\in j_{i}\left(A_{\kappa}\right)$. If $\mathscr{U}_{i+1} \in M_{i+1}$ is a normal measure over $\kappa_{i+1}$ so that $M_{i+2}=$ transitive collapse $\left(M_{i+1}^{\kappa_{i+1}} / \mathscr{U}_{i+1}\right) \vDash$ " $\kappa_{i+1}$ is non-measurable" $\left(\mathscr{U}_{i+1}\right.$ exists since $j_{i}\left(A_{\kappa}\right) \in M_{i+1}$ is a set of measurable cardinals), then let $j_{i+1}: M_{i+1} \rightarrow M_{i+2}$ be the associated elementary embedding. Note that as $j_{i+1}\left(\kappa_{l}\right)=\kappa_{l}$ for $i+2 \leq$ $l \leq n, M_{i+2} \vDash$ " $\kappa_{i+1}$ is non-measurable and $j_{i+1}\left(\kappa_{l}\right)=\kappa_{l}$ is measurable for $i+2 \leq l \leq n$ ". We can thus inductively for $i+2 \leq l \leq n$ find a normal measure $\mathscr{U}_{l} \in M_{l}$ so that $M_{l+1}=$ transitive collapse $\left(M_{l}^{\kappa_{l}} / \mathscr{U}_{l}\right) \vDash " \kappa_{i+1}, \ldots, \kappa_{l}$ are nonmeasurable and $\kappa_{l+1}, \ldots, \kappa_{n}$ are measurable". This is since the associated elementary embedding $j_{l}: M_{l} \rightarrow M_{l+1}$ is so that $j_{l}\left(\kappa_{m}\right)=\kappa_{m}$ for $i+1 \leq$ $m<l$ as $\kappa_{l}$ is the critical point of $j_{l}$, inductively, $M_{l} \vDash " \kappa_{i+1}, \ldots, \kappa_{l-1}$ are non-measurable", and $\mathscr{U}_{l}$ has been chosen so that $M_{l+1} \vDash$ " $\kappa_{l}$ is nonmeasurable"; also, as above, $j_{l}\left(\kappa_{m}\right)=\kappa_{m}$ for $l+1 \leq m \leq n$, and we can inductively assume $M_{l} \vDash$ " $\kappa_{l+1}, \ldots, \kappa_{n}$ are measurable". If we now define $j=j_{n} \circ j_{n-1} \circ \cdots \circ j_{i+1} \circ j_{i}$ and $M=M_{n+1}$, then it can be verified that $j: V \rightarrow M$ is an elementary embedding witnessing the $\lambda$ strong compactness of $\kappa$. By construction, $M \vDash$ " $\kappa_{i+1}, \ldots, \kappa_{n}$ are non-measurable", so there is therefore no problem (as the forcing creates no new measurable cardinals) if $G$ is $V$-generic over $P^{\kappa}$ in finding $H \subseteq j\left(P^{\kappa}\right), H \in V[G]$ so that $j$ extends to $j^{\prime}: V[G] \rightarrow M[G * H]$, which is a strongly compact embedding.

Unfortunately, however, the above iteration breaks down at stage $\omega$, as we have to consider new $\omega$ sequences, and our control over $j\left(P^{\kappa}\right)$ may be lost. To this point in time, no way is yet known around this difficulty, and the KimchiMagidor method is applicable only in situations dealing with a finite number of cardinals. In particular, in our situation, we of course do not destroy all measurable cardinals, only those cardinals $\delta$ forced to be $\varphi_{\kappa}(\delta)$ supercompact, which as we have previously observed, are those cardinals $\delta$ actually $\varphi_{\kappa}(\delta)$ supercompact in $V$. The argument we use to show $\kappa$ remains strongly compact is then essentially the same as the one just given. The only real difference is that in order to preserve the strong comapctness and $\varphi_{\kappa}\left(\kappa_{l}\right)$ supercompactness of $\kappa_{l}$ for $l=i+1, \ldots, n$, we choose for $i+1 \leq l \leq n$ the measures $\mathscr{U}_{l}$ to be supercompact measures over the appropriate version of $P_{\kappa_{l}}\left(\varphi_{\kappa}\left(\kappa_{l}\right)\right)$ with corresponding elementary embeddings $j_{l}: M_{l} \rightarrow M_{l+1}$ so that $M_{l+1} \vDash{ }^{\text {" }} \kappa_{m}$ for $i+1 \leq m \leq l$ isn't $\varphi_{\kappa}\left(\kappa_{m}\right)$ supercompact and $\kappa_{m}$ for $l+1 \leq m \leq n$ is $\varphi_{\kappa}\left(\kappa_{m}\right)$ supercompact" and then define $j$ as before. Unfortunately, this doesn't eliminate the difficulty at stage $\omega$, and we can still only handle a finite number of cardinals.

The above paragraph illustrates why restriction (c) of the statement of the Theorem is necessary, i.e., why we must have $\varphi_{\kappa_{i}}(\delta) \geq \varphi_{\kappa_{j}}(\delta)$ if $i \leq j$ and $\delta$ is both $\varphi_{\kappa_{i}}(\delta)$ and $\varphi_{\kappa_{j}}(\delta)$ supercompact in $V$. If, e.g., $\varphi_{\kappa_{i}}$ were the function $\delta \mapsto \delta^{+}, \varphi_{\kappa_{i+1}}$ were the function $\delta \mapsto \delta^{++}$, and $V \vDash " 2^{\kappa_{i+1}^{+}}=\kappa_{i+1}^{++}$", then in $V^{Q_{\kappa_{i}}}$ (we use the notation of Lemma 4) we would have unboundedly many cardinals $\delta$ in the interval $\left(\kappa_{i}, \kappa_{i+1}\right)$ which were $\delta^{+}$supercompact. As was just noted, when forcing with $P^{\kappa_{i}}$, we can only currently preserve the $\delta^{+}$supercompactness of finitely many such $\delta$ if $\kappa_{i}$ is to remain strongly compact. If we destroy the $\delta^{+}$supercompactness of all but finitely many of these $\delta$, then $\kappa_{i+1}$ will no longer be $\kappa_{i+1}^{++}$supercompact, although the strong compactness of $\kappa_{i}$ will be able to be preserved. Of course, if $\varphi_{\kappa_{i}}$ were the function $\delta \mapsto \delta^{++}$, $\varphi_{\kappa_{i+1}}$ were the function $\delta \mapsto \delta^{+}$, and $V \vDash{ }^{\prime} 2^{\kappa_{i+1}^{+}}=\kappa_{i+1}^{++}$", then in $V^{Q_{\kappa_{i}}}$, there 
would be no cardinals $\delta$ which were $\delta^{+}$or $\delta^{++}$supercompact in the interval $\left(\kappa_{i}, \kappa_{i+1}\right)$, thereby eliminating this difficulty.

In conclusion, as the referee has pointed out, there is an interesting comparison the reader can make between the result of this paper and the result of $[\mathrm{KM}]$. Although the arguments we give in general follow the proof of the result that from $n \in \omega$ supercompact cardinals, it is possible to force and obtain the coincidence of the first $n$ strongly compact cardinals with the first $n$ measurable cardinals, the forcing notion itself is similar to the one used in the proof of the result that from a class of supercompact cardinals, it is possible to force and obtain the coincidence of the classes of strongly compact and supercompact cardinals (except at limit points).

\section{ACKNOWLEDGMENT}

The author wishes to acknowledge helpful conversations with Yechiel Kimchi, Menachem Magidor, and Saharon Shelah on the subject matter of this paper and on related topics. In addition, the author wishes to acknowledge gratefully the referee for pointing out the gap in the proofs of both the original version of this paper and the corresponding result of $[\mathrm{KM}]$ and for making a number of extremely useful comments. Many of the referee's remarks have been incorporated into the body of this paper. In particular, most of the concluding comments above are due almost verbatim to the referee.

\section{REFERENCES}

[A1] A. Apter, On the least strongly compact cardinal, Israel J. Math. 35 (1980), 225-233.

[A2] - Some results on consecutive large cardinals, Ann. Pure Appl. Logic 25 (1983), 1-17.

[KM] Y. Kimchi and M. Magidor, The independence between the concepts of strong compactness and supercompactness (in preparation).

[KP] K. Kunen and J. Paris, Boolean extensions and measurable cardinals, Ann. Math. Logic 2 (1971), 359-378.

[L] R. Laver, Making the supercompactness of $\kappa$ indestructible under $\kappa$-directed closed forcing, Israel J. Math. 29 (1978), 385-388.

[LS] A. Lévy and R. Solovay, Measurable cardinals and the continuum hypothesis, Israel J. Math. 5 (1967), 234-248.

[Ma] M. Magidor, How large is the first strongly compact cardinal?, Ann. Math. Logic 10 (1976), 33-57.

[Me] T. Menas, On strong compactness and supercompactness, Ann. Math. Logic 7 (1975), 327-360.

[SRK] R. Solovay, W. Reinhardt, and A. Kanamori, Strong axioms of infinity and elementary embeddings, Ann. Math. Logic 13 (1978), 73-116.

Department of Mathematics, Baruch College of City University of New York, New YORK, NEW YORK 10010

E-mail address: awabbecunyvm. cuny.edu 